\title{
Large voltage from spin pumping in magnetic tunnel junctions
}

\author{
S. T. Chui, Z. F. Lin \\ Bartol Research Institute and Department of Physics and Astronomy, \\ University of Delaware, Newark, DE 19716
}

(Dated: October 25, 2018)

\begin{abstract}
We studied the response of a ferromagnet-insulator-normal metal tunnel structure under an external oscillating radio frequency (R.F.) magnetic field. The D. C. voltage across the junction is calculated and is found not to decrease despite the high resistance of the junction; instead, it is of the order of $\mu V$ to $100 \mu V$, much larger than the experimentally observed value (100 nano-V) in the "strong coupled" ohmic ferromagnet-normal metal bilayers. This is consistent with recent experimental results in tunnel structures, where the voltage is larger than $\mu V \mathrm{~s}$. The damping and loss of an external RF field in this structure is calculated.
\end{abstract}

PACS numbers: PACS numbers:73.40.-c,71.70.Ej,75.25.+z 
There has been much recent interest in the spin dynamics in hybrid nanostructures composed of ferromagnetic and normal metal layers.[1-5] Following earlier work on the spin torque effect, the spin pumping effect [6] has been demonstrated for "strongly coupled" ohmic metallic multilayers as an additional contribution to the FMR linewidth in FM/NM multilayers (where $\mathrm{NM}$ is $\mathrm{Pt}, \mathrm{Pd}, \mathrm{Cu}$, etc.) [7] and more recently as a difference in voltages of the order of $100 \mathrm{nV}$ between two FM/NM interfaces of a NM1/FM/NM2 trilayer. [8] Two types of metallic structures are commonly studied. In addition to the strongly coupled ohmic multilayer systems, weakly coupled tunnel structures have also been extensively studied. The physics of these two types of systems can be very different. [9] In particular, for the tunnel structures, the coupling of the longitudinal magnetization and the charge lead to magnetization and charge dipole layers at the interface. After the effect of the electron-electron interaction is included, it is found that because of the large difference of the length scales associated with the charge (screening length, $\approx 1 \AA$ ) and the spin (spin diffusion length, $\approx 100 \AA$ ) fluctuations, there is a larger splitting of the chemical potentials than that predicted by the conventional spin accumulation picture.[10] Whereas the conventional picture suggests that the splitting scales with the current and will decrease with an increase with resistance, this is no longer true in the more complete picture.

Recently Moriyama and coworkers [11] reported measurements of the dc voltage attributed to the spin pumping effect in different tunnel junctions, and demonstrate that the voltage is larger than micro-volts, enhanced orders of magnitude compared to that for metallic trilayers. In this paper, we generalize our recent work on spin torque[13] to the spin pumping situation and found an enhanced voltage for the tunnel structures, in agreement with the experimental results. We now describe our results in detail.

The system we have in mind is a ferromagnet-normal metal tunnel junction where the two interfaces between the ferromagnet-insulator-metal sandwich structure are assumed to be at $z= \pm d / 2$. We assume the $z$ axis to be perpendicular to the faces of the tunnel junction. The initial magnetization is assumed to be in the $x-y$ plane with an orientation given by $\mathbf{p}_{0}^{L}=\mathbf{e}_{x}$ for the ferromagnet on the left hand side of the sandwich structure.

Because the work functions of the metals on opposite sides of the junction may not be equal, at zero external radio frequency (RF) field there will be a charge dipole layer formed at the interfaces. What we are calculating here are the changes from the zero field situation. This surface inhomogeneity can lead to an additional contribution to the increase in the 
FMR damping, as we explain below. The experimental structures usually possess edge domains where the switching starts. The magnetization is thus not completely uniform in the $x-y$ plane. To bring out the essential physics, we shall not consider this complication in the present paper but we hope to come back to this in the future.

Under an external time varying RF field, we expect the magnetization in the ferromagnet to be a sum of a uniform magnetization which is a solution of the inhomogeneous Bloch (Landau-Gilbert) equation due to the external field and a spatially varying solution of the homogeneous equation so that the boundary condition can be satisfied. This spatially varying part provides for the additional damping and the voltage observed in the experiments. Our approach is to obtain general solutions in each part of the junction (Eq. (11), (12), (15), (16)). The amplitudes of these solutions are determined by the boundary conditions (Eq. (7)). From these amplitudes, the voltage and the damping can be derived. We first describe the general solution of the magnetization in a ferromagnet.

Magnetization in a ferromagnet: Our starting point is the equation of motion of the charge and the magnetization. For the charge, it is just the equation of charge current conservation

$$
\nabla \cdot \mathbf{J}_{e}=-\frac{\partial \delta n}{\partial t}
$$

where $\mathbf{J}_{e}$ is the total current. The equation for the magnetization $\mathbf{M}$ has been much discussion extensively in the past.[12] The equation takes the form of the phenomenlogical classical Landau-Lifshitz (Bloch) equation with longitudinal and transverse damping and an additional source term

$$
\frac{\partial \mathbf{M}}{\partial t}-\gamma \mathbf{M} \times \mathbf{H}-\alpha \mathbf{M} \times(\mathbf{M} \times \mathbf{H})+\nabla \cdot \hat{\mathbf{J}}_{\mathbf{M}}=-\frac{\delta \mathbf{M}}{\tau}
$$

where $\gamma$ is the gyromagnetic ratio, and $\mathbf{H}$ is the effective field describing the precession of the magnetic moments given by $\mathbf{H}=\mathbf{H}_{\mathrm{e}}+\mathbf{H}_{\mathrm{an}}+\mathbf{H}_{\mathrm{dip}}+\mathbf{H}_{\mathrm{ex}} . \mathbf{H}_{\mathrm{ex}}=J \nabla^{2} \mathbf{M}$ is the effective field due to direct exchange; the anisotropy term includes a bulk and a surface anisotropy energy $\mathbf{H}_{\mathrm{an}}=H_{a b}+H_{a s}, H_{a b}=K \mathbf{M}_{0}, H_{a s}=K_{s} M_{s}$ where $M_{s}=\mathbf{M}_{0} \delta(z+d / 2)$. Here we have separated a bulk and a surface contribution that acts on the surface magnetization $M_{s}$. For simplicity we have assumed this surface contribution localized at the interface. The other terms can also contain a surface contribution and can be treated in a similar manner as this ansiotropy contribution. For simplicity of presentation we illustrate our results with just this term. $\mathbf{H}_{\mathrm{e}}$ represents the external field; and $\mathbf{H}_{\text {dip }}$ denotes the dipole-dipole interaction. 
$\hat{\mathbf{J}}_{\mathrm{M}}$ is a spin current (tensor). The currents are driven by density gradients (diffusion) and external forces.

$$
\begin{aligned}
& \mathbf{J}_{e}=-\sigma \nabla V-e D \nabla \delta n-D_{M} \nabla\left(\Delta \mathbf{M} \cdot \mathbf{p}_{0}\right) \\
& \hat{\mathbf{J}}_{\mathrm{M}}=-\sigma_{M} \nabla\left(V \mathbf{p}_{0}\right)-D_{M}^{\prime} \nabla \Delta \mathbf{M}-D^{\prime} \nabla\left(\delta n \mathbf{p}_{0}\right)
\end{aligned}
$$

where $\sigma, \sigma_{M}$ are the effective conductivities for the charge and magnetization. $\mathbf{p}_{0}$ is a unit vector along the direction of the equilibrium magnetization: $\mathbf{p}_{0}=\frac{\mathbf{M}_{0}}{\left|\mathbf{M}_{0}\right|}$ with $\mathbf{M}_{0}$ the local equilibrium magnetization, $\Delta \mathbf{M}=\mathbf{M}\left(1-|\mathbf{M}| / M_{0}\right)$ is a change in magnetization. $D, D^{\prime}$, $D_{M}, D_{M}^{\prime}$ are the effective diffusion constants. $V=V_{e}+W$, with $V_{e}$ the electric potential describing the external electric field and $W$ the local electric (screening) potential due to the other electric charges determined self-consistently by

$$
W(\mathbf{r})=\int d^{3} \mathbf{r}^{\prime} U\left(\mathbf{r}-\mathbf{r}^{\prime}\right) \delta n\left(\mathbf{r}^{\prime}\right)
$$

with $U$ the Coulomb potential. The total number density of charge carriers and $x$ component of magnetization are given by $n=\sum_{s} n_{s}, M_{x}=\sum_{s} s n_{s}$. In the coordinate system with one of the coordinate axis along the direction of the magnetization, the spin current can be understood as the difference of the spin up current and the spin down current. The vector dependence is such that the equation is covariant. The Landau-Liftshitz equation without the source term $\nabla \cdot J_{M}$ is believed to describe the physics of ordinary domain walls where the direction of the magnetization changes but its magnitude remains fixed. Eq. (3) is consistent with this belief. For ordinary domain walls, $J_{M}=0 . \tau$ is the longitudinal relaxation time, describing the relaxation of the system towards its local equilibrium value of magnetization. $\alpha$ measures the transverse (Gilbert) damping term.

Substituting the expression for $\hat{\mathbf{J}}_{\mathrm{M}}$ into the modified Landau-Lifshitz equation (2) we obtain the linearized relaxation equation for $\mathbf{M}$ :

$$
\begin{gathered}
\nabla^{2} \delta \mathbf{M}-\left(\frac{1}{l_{s f}^{2}}+i \omega / D_{M}^{\prime}\right) \delta \mathbf{M}+\zeta \mathbf{p}_{0} \times\left(\nabla^{2} \delta \mathbf{M}-\frac{\kappa}{l_{s f}^{2}} \delta \mathbf{M}-\kappa_{s} \delta \mathbf{M}_{s} / l_{s f}^{2}\right)+\gamma\left(\delta \mathbf{M} \times \mathbf{H}_{0}+\mathbf{M}_{0} \times \mathbf{H}_{1}\right) \\
+\alpha \delta \mathbf{M}_{\perp} M_{0} H_{0} / D_{M}^{\prime}=-\left(D^{\prime} / D_{M}^{\prime}\right) \mathbf{p}_{0}\left(\nabla^{2} \delta n-\frac{\delta n}{\lambda_{0}^{2}}\right)
\end{gathered}
$$

where only $\mathbf{H}_{\text {ex }}=J \nabla^{2} \mathbf{M}=J \nabla^{2} \delta \mathbf{M}$ and $\mathbf{H}_{\text {an }}=K \mathbf{M}_{0}$ are kept in the precession term $\gamma \mathbf{M} \times \mathbf{H}$, and use has been made of Gauss' law: $\nabla^{2} V=\nabla^{2} W=-\frac{e}{\epsilon_{0}} \delta n$. The bare spin diffusion length $l_{s f}$ and the bare screening length $\lambda_{0}$ are given by $l_{s f}^{2}=\tau D_{M}^{\prime}$ and $\lambda_{0}^{2}=\frac{\epsilon_{0} D^{\prime}}{\sigma}$ respectively. Other dimensionless parameter are $\zeta=\frac{\gamma\left|\mathbf{M}_{0}\right| J}{D_{M}^{\prime}}$ and $\kappa=\frac{l_{s f}^{2} K}{J}$. 
The charge current conservation (1) yields, for the steady state without linearization,

$$
\frac{1}{\lambda_{0}^{2}} \delta n-\left(\nabla^{2}-i \omega / D\right) \delta n-\left(D_{M} / D\right) \nabla^{2}\left(\delta \mathbf{M} \cdot \mathbf{p}_{0}\right)=0
$$

which, together with Eq.(15), describes the distribution of the charge and magnetization away from the tunnel junction in terms of their values at the junction. (To simplify the algebra, we have made the approximation that $\left.\mathrm{D}=\mathrm{D}^{\prime}\right)$. The values of the charge and magnetization densities at the junction can be determined by matching boundary conditions across the barrier. We first solve these equations in the metal part of the junction. These solutions determine the charge and magnetization dipole layers.

The solution of eq. (5) can be written a sum of two terms,

$$
\delta \mathbf{M}=\delta \mathbf{M}_{0}+\delta \mathbf{m}^{i}
$$

a spatially uniform $\left(\delta \mathbf{m}^{i}\right)$ solution of the bulk inhomogeneous equation with the source term $\mathbf{M}_{0} \times \mathbf{H}_{1}$ and a sptially varying solution $\left(\delta \mathbf{M}_{0}\right)$ of the homogeneous equation. The inhomogeneous bulk equation is

$$
-\left(\frac{1}{l_{s f}^{2}}+\left(i \omega-\alpha^{\prime}\right) / D_{M}^{\prime}\right) \delta \mathbf{m}-\zeta \mathbf{p}_{0} \times \frac{\kappa}{l_{s f}^{2}} \delta \mathbf{m}+\gamma\left(\delta \mathbf{m} \times \mathbf{H}_{0}+\mathbf{M}_{0} \times \mathbf{H}_{1}\right)=0
$$

where $\alpha^{\prime}=\alpha M_{0} H_{0}$. This is the conventional FMR equation, which can be readily solved. Define $\mathbf{e}_{ \pm}=\mathbf{e}_{z} \pm i \mathbf{e}_{y}$, then $\mathbf{e}_{x} \times \mathbf{e}_{ \pm}= \pm i \mathbf{e}_{ \pm}$. We write the transverse magnetization as $\delta \mathbf{m}_{\perp}^{i}=\sum \delta m_{ \pm} \mathbf{e}_{ \pm}$and obtain

$$
\delta m_{ \pm}=\chi_{ \pm}^{0} H_{1, \pm}
$$

where $1 / \chi_{ \pm}^{0}=\left[\left( - \pm i \frac{1}{l_{s f}^{2}}+\left(i \omega-\alpha^{\prime}\right) / D_{M}^{\prime}\right)+\zeta \frac{\kappa}{l_{s f}^{2}}+\gamma H_{0}\right] / \gamma M_{0}$. Associated with this transverse magnetization, there is a change of the longitudinal magnetization given by

$$
\delta m_{x}^{i}=M_{0}-\left(M_{0}^{2}-\delta m_{\perp}^{i 2}\right)^{1 / 2} \approx 0.5 \delta m_{\perp}^{i 2} / M_{0}
$$

This is the lowest order correction to the longitudinal magnetization. Higher order nonlinear corrections to the transverse magnetzation will produce changes in the longitudinal component that is higher than $3 r d$ order in $H_{1}$. In the equation of motion (2), no lower order correction are produced.

The equation for the spatially varying term becomes

$\nabla^{2} \delta \mathbf{M}_{\mathbf{0}}-\left(\frac{1}{l_{s f}^{2}}+i \omega / D_{M}^{\prime}\right) \delta \mathbf{M}_{\mathbf{0}}+\zeta \mathbf{p}_{0} \times\left[\nabla^{2} \delta \mathbf{M}_{\mathbf{0}}-\frac{\kappa}{l_{s f}^{2}} \delta \mathbf{M}_{\mathbf{0}}-\kappa_{s}\left(\delta \mathbf{m}^{i}+\delta \mathbf{M}_{0 s}\right) / l_{s f}^{2}\right]+\gamma \delta \mathbf{M}_{0} \times \mathbf{H}_{0}$ 


$$
+\alpha \delta \mathbf{M}_{0 \perp} M_{0} H_{0} / D_{M}^{\prime}=-\left(D^{\prime} / D_{M}^{\prime}\right) \mathbf{p}_{0}\left(\nabla^{2} \delta n-\frac{\delta n}{\lambda_{0}^{2}}\right)
$$

The solution of this equation is similar to that in our previous studies. [13] Away from the boundary, the surface terms are absent. This equaion becomes homogeneous. We solve this homogeneous equation and form linear combinations of them to satisfy the boundary constraints. By integrating this equation over a small region of space at the boundary we arrive at the condition that the difference between the tunnelling and the ferromagnet pseudo spin current is equal to surface anisotropy term:

$$
J_{\mathbf{M}}^{t}-I_{\mathbf{M}}^{L}=\gamma K_{s} \mathbf{M}_{0} \times\left(\delta \mathbf{m}^{i}+\delta \mathbf{M}_{0 s}\right) .
$$

where $I_{\mathbf{M}}^{t}$ is the tunnelling magnetization current, the pseudo spin current $[14] J_{\mathbf{M}}=J_{\mathbf{M}}-$ $\gamma J \mathbf{M}_{0} \times \partial_{z} \delta \mathbf{M}$ includes an extra term involving the exchange that affects only the transverse magnetization current. We expect this extra term to be also present for ohmic junctions but so far it has not been included. In previous spin pumping studies on ohmic junctions, a term of a similar functional form $g \mathbf{n} \times \partial \mathbf{n} / \partial t(\mathbf{n}=\mathbf{M} /|M|)$ has been discussed. However, the coefficient was interpreted as a spin mixing conductance. We next discuss the solution of the homogeneous equation.

We expect the charge and magnetization dipole layers to decay away from the interface with length scales controlled by the spin diffusion length and the screening length. Because of the vector nature of the magnetization, there are three normal modes by which they can decay away from the interface. Including the charge degree of freedom, there are four normal modes that one can consider. For the ferromagnetic metal on the left hand side, we thus consider the following ansatz:

$$
\delta n^{L}=\sum_{i=1}^{4} \delta n_{i 0}^{L} e^{\left(z+\frac{d}{2}\right) / l_{i}}, \quad \delta \mathbf{M}_{0}^{L}=\sum_{i=1}^{4} \delta \mathbf{M}_{i 0}^{L} e^{\left(z+\frac{d}{2}\right) / l_{i}},
$$

where the superscript $L$ denotes the left hand side.

Letting the coefficients before the exponential scaling functions vanish for steady-state solutions, we get for small $\omega$ the renormalized screening length

$$
l_{1}=\lambda_{0} \xi_{1}^{1 / 2}
$$

the renormalized spin diffusion length

$$
l_{2}=l_{s f} \xi_{2}^{1 / 2}
$$


and a combination of the exchange length and the spin diffusion length

$$
l_{3,4}=l_{s f} /\left[\left(1- \pm i \zeta \kappa_{r}\right) /(1- \pm i \zeta)+\left(i \omega-\alpha^{\prime}\right) l_{s f}^{2} /\left(D_{M}^{\prime 2}(1- \pm i \zeta)\right)\right]^{1 / 2} .
$$

The $\xi$ s and $\beta$ are measures of the asymmetry of the spin up and spin down conductivities of the ferromagnet: $\xi_{1}=\left[1-D^{\prime} D_{M} /\left(D D_{M}^{\prime}\right)\right] /\left[1-\sigma_{M} D^{\prime} D_{M} /\left(\sigma D D_{M}^{\prime}\right)+i \omega \lambda_{0}^{2} / D\right] . \xi_{2}=$ $\left(1-\beta^{2}\right) /\left(1-i \omega l_{s f}^{2} / D_{M}^{\prime}\right), \beta^{2}=\left[1-D^{\prime} D_{M} \sigma_{M} /\left(\sigma D D_{M}^{\prime}\right)\right] . \kappa_{r}=\kappa+\gamma H_{0} l_{s f}^{2} / \zeta M_{0}$. As we shall see below, $l_{3}$ and $l_{4}$ correspond to length scales with which the "precession" dies away from the interface. The additional term $\gamma \delta M \times H_{0}$ modifies these two lengths accordingly. The screening length and the spin diffusion length are renormalized. From eq. (6) we find that the charge densities can be related to the magnetization densities by

$$
\delta n_{10}^{L}=e\left(\xi_{1}^{L}-1\right) \delta M_{10}^{L} / \mu_{B}, \quad \delta n_{20}^{L}=\frac{e \lambda_{0}^{2} D_{M}^{L}}{\mu_{B} l_{2}^{2} D^{L}} \delta M_{20}^{L}, \quad \delta n_{30}^{L}=\delta n_{40}^{L}=0 .
$$

Because $l_{2} \gg \lambda_{0}, \delta n_{20} / e<<\delta M_{20} / \mu_{B}$. As we see below, in general $\delta M_{20}$ is much less than $\delta M_{10}$. Inserting the "eigen-solutions" into equations (요), we finally obtain analytic expressions for the dipole layers:

$$
\begin{aligned}
\delta n^{L} & =\delta n_{10}^{L} e^{\left(z+\frac{d}{2}\right) / l_{1}}+\delta n_{20}^{L} e^{\left(z+\frac{d}{2}\right) / l_{2}} \\
\delta \mathbf{M}^{L} & =\mathbf{p}_{0}^{L} \delta M_{10}^{L} e^{\left(z+\frac{d}{2}\right) / l_{1}}+\mathbf{p}_{0}^{L} \delta M_{20}^{L} e^{\left(z+\frac{d}{2}\right) / l_{2}}+\mathbf{e}_{+}^{L} \delta M_{30}^{L} e^{\left(z+\frac{d}{2}\right) / l_{3}}+\mathbf{e}_{-}^{L} \delta M_{40}^{L} e^{\left(z+\frac{d}{2}\right) / l_{4}}
\end{aligned}
$$

The two transverse modes corresponds to the left and right circularly polarized modes $\mathbf{e}_{ \pm}$. $\delta M_{i 0}^{L}$, with $i=1,2,3,4$, are to be determined later. Terms of the order $\left(\lambda_{0} / l_{s f}\right)^{2}$ or higher have been neglected since $l_{s f}^{2}>>\lambda_{0}^{2}$. Also, to simplify the algebra we have assumed that the ferromagnetic thickness $d_{F}$ to be larger than the spin diffusion length so that we do not need to worry about "reflection" effects from the leads. As advertised, the charge dipole layer is the sum of two terms, one decaying with a length scale of the screening length; the other, the spin diffusion length. The vector magnetization dipole is now a sum of four terms. The first two ( $\left.\delta \mathbf{M}_{10}^{L}, \delta \mathbf{M}_{20}^{L}\right)$ are along the direction of the original magnetization; the last two are perpendicular to the direction of the original magnetization and describes the precession of the magnetization around the original axis. Again, the first two terms correspond to decay lengths of the order of the spin diffusion length and the screening length, while the precession term only decays with a length scale that is a combination of the exchange length and the spin diffusion length. 
With equations (11) and (12), the charge and magnetization currents $\mathbf{J}_{e}$ and $\hat{\mathbf{J}}_{\mathrm{M}}$ can be worked out as

$$
\begin{aligned}
\mathbf{J}_{e}^{L}= & \sigma \mathbf{E}_{e x t} \\
\hat{\mathbf{J}}_{\mathrm{M}}^{L}= & \sigma_{M} \mathbf{E}_{e x t} \mathbf{p}_{0}^{L} / e+ \\
& +\frac{\left(1-\beta^{2}\right) D_{M}^{\prime}}{l_{2}} \mathbf{e}_{z} \mathbf{p}_{0}^{L} \delta M_{20}^{L} e^{\delta z / l_{2}}+\frac{D_{M}^{\prime}}{l_{3}} \mathbf{e}_{z} \mathbf{e}_{+} \delta M_{30}^{L} e^{\delta z / l_{3}}+\frac{D_{M}^{\prime}}{l_{4}} \mathbf{e}_{z} \mathbf{e}_{-} \delta M_{40}^{L} e^{\delta z / l_{4}}
\end{aligned}
$$

where $\delta z=z+\frac{d}{2}, \mathbf{E}_{e x t}=E_{e x t} \mathbf{e}_{z}$ is the external electric field inside the conductor. Note that the magnetization current is not a function of the rapidly varying part of the charge and magnetization densities $\delta n_{10}, \delta M_{10}$. In principle, the magnetization current can contain a term of the form $J_{M 1} \exp \left(z / l_{1}\right)$. In the generalized Landau-Gilbert equation (Eq. (2)), terms of different functional dependence are each equal to zero. The only terms that are proportional to $\exp \left(z / l_{1}\right)$ comes from $\nabla \cdot J_{M}$ and is proportional to $J_{M 1} / l_{1}$. This term and hence its contribution to the magnetization current is equal to zero. To match the quantities at the boundaries we next consider the charge and magnetization in a normal metal $(\mathrm{N})$.

Normal metal: On the N side, the charge and magnetization are not coupled. The charge is given by

$$
\delta n^{R}=\delta n_{0}^{R} \exp (-z / \lambda)
$$

The magnetization satisfies the equation $\partial_{t} \mathbf{M}=\left(D_{n} \partial_{z}^{2}-1 / \tau_{s f}^{N}\right) \mathbf{M}=0$. From this we obtain

$$
\delta \mathbf{M}^{R}=\delta \mathbf{M}_{0}^{R} \exp \left[-(z-d / 2) / l_{R}\right]
$$

The longitudinal magnetization current at the interface $(\mathrm{z}=\mathrm{d} / 2)$ is given by $J_{M}^{R}=$ $-D_{N} \delta M_{0 x}^{R} / l_{R}$

The longitudinal magnetization current at the left interface is given by eq. (16). Equating $J_{M}^{R}$ to $J_{M}^{L}$, we get

$$
\delta M_{0 x}^{R}=-\left(1-\beta^{2}\right) D_{M}^{\prime L} \delta M_{20}^{L} l_{R} /\left[D_{N} l_{2}\right]
$$

The magnetization on the right is proportional to $\delta M_{20}^{L}$ and is not a function of $\delta M_{10}^{L}$. As we shall see below, $\delta M_{10}^{L}>>\delta M_{20}^{L}$, hence the longitudinal magnetization change on the right is much less than that on the left at the boundary. The charge neutrality condition $\int_{\frac{d}{2}}^{\infty} \delta n^{L} d z+\int_{-\infty}^{-\frac{d}{2}} \delta n^{L} d z=0$ yields

$$
\delta n_{0}^{R}=-\left(l_{1} \delta n_{10}^{L}+l_{2} \delta n_{20}^{L}\right) / \lambda,
$$


These two equations express the quantities on the right in terms of quantities on the left. We now determine the amplitudes of these physical quantities by matching the boundary condition as in eq. (7).

Boundary conditions: The longitudinal magnetization current in the ferromagnet arriving at the interface $I_{M}^{L}$ is equal to the magnetization current $J_{M}^{t}$ across the interfacce due to tunnelling because the term on the right hand side of eq. (7) is along the transverse direction. For the longitudinal component, $I_{m}=J_{m}$. The longitudinal magnitization tunnelling current is equal to the difference of the spin up and the spin down tunnelling current. From standard calculations of the tunnelling current[15] we get

$$
J_{M}^{t}=\sum_{s} s\left|T_{s s^{\prime}}\right|^{2}\left(\delta n_{L s}\left(E+\delta \mu_{s}^{L}\right)-\delta n_{R s}\left(E+\delta \mu_{s}^{R}\right)\right)
$$

Here $\delta \mu$ contains contributions from the electric potential due to the charges at the interface and that from the accumulation due to the bottleneck effect. The change of the electron density of spin s can be related to the change of the total charge and magnetization densities by (we use units so that $\mu_{B}=1$ ): $\delta n_{s}=0.5\left(\delta n+s \delta M_{x}\right)$. The longitudinal magnetization density is the sum of contributions from the solutions of the homogeneous and the inhomogeneous equations:

$$
\delta M_{x}=\delta m_{x}^{i}+\delta M_{x 0}
$$

From eq. (14) $J_{M}^{L}=\frac{\left(1-\beta^{2}\right) D_{M}^{\prime}}{l_{2}} \delta M_{20}^{L}$. The inhomgeneous term $\delta m^{i}$ is uniform and does not contribute to the magnetization current $J_{M}^{L}$ inside the ferromagnet. From $J_{M}^{t}=J_{M}^{L}$, we get

$$
\left(1-\beta^{2}\right) D_{M}^{L} \delta M_{20} / l_{2}=\sum_{s} s\left|T_{s s}\right|^{2}\left(\delta n_{L s}-n_{R s}\right) .
$$

All variables of this equation can be written in terms of the two independent variables $\delta M_{10,20}$. Now $\delta n_{0 L s}=0.5\left[\delta n_{10, L}+\delta n_{20, L}+s\left(\delta M_{10, L}+\delta M_{20, L}\right).\right]$ Using eq. (11) and (16) we get

$$
\left(1-\beta^{2}\right) D_{M}^{\prime L} \delta M_{20} / l_{2}=\sum_{s} s\left|T_{s s}\right|^{2}\left[\delta M_{10}^{L}\left(\xi^{L}-1+s+l_{1}\left(\xi^{L}-1\right) / \lambda\right)+s \delta m_{x}^{i}\right] .
$$

This equation implies that $\delta M_{20}$ is of the order of $c_{t} \delta M_{10}^{L} / c_{m}$ where $c_{t}\left(c_{m}\right)$ is the tunnelling (metallic) conductance. $c_{t}$ much smaller than the metal conductance $c_{m}$. Thus $\delta M_{20}$ is much smaller than $\delta M_{10}$.

For an open circuit, the total charge tunnelling current is zero. We get $J=$ $\sum_{s}\left|T_{s s}\right|^{2}\left(\delta n_{L s}-n_{R s}\right)=0$. Substituting in the expresssions for the charge densities and 
using the condition that $\delta M_{20}<<\delta M_{10}$, we get

$$
\sum_{s}\left|T_{s s}\right|^{2}\left[\delta M_{10}^{L}\left(\xi^{L}-1+s+l_{1}\left(\xi^{L}-1\right) / \lambda\right)+s \delta m_{x}^{i}\right]=0 .
$$

Solving this equation, we finally obtain

$$
\delta M_{10}^{L}=-f \delta m_{x}^{i}
$$

where $f=\left(\sum_{s}\left|T_{s s}\right|^{2} s\right) /\left[\sum_{s}\left|T_{s s}\right|^{2}\left(\xi^{L}-1+s+l_{1}\left(\xi^{L}-1\right) / \lambda\right)\right]$. The corresponding charge is, from eq. (10), $\delta n_{10}^{L}=\left(\xi^{L}-1\right) f \delta m_{x}^{i}$. The charge and the magnetization densities are proportional only to the ratio of the conductances. Hence they are not necessarily small for tunnel junctions. As we emphasized before[9], this comes about because $\lambda<<l_{s f}$.

Emf:

The DC voltage is estimated as the change of the mean chemical potential across the interface, given by $\Delta V=0.5 \sum_{s} \Delta\left(\delta \mu_{s}\right)=0.5 \sum_{s} \Delta\left(\delta n_{s} / N_{s}\right)$ where $N_{s}$ is the density of states. This drop includes a contribution from an electric potential as well as a contributions from electron density changes due to bottleneck and electron-electron interaction effects. This drop can be written as $\left(\delta M^{L}>>\delta M^{R}\right)$

$$
\Delta V=0.25\left(e \delta m_{x}^{i} / \mu_{B}\right) f\left[\left(\xi^{L}-1\right)\left(1 / N_{+}^{L}+2 l_{1} /\left(\lambda^{R} N^{R}\right)+1 / N_{-}^{L}\right)+1 / N_{+}^{L}-1 / N_{-}^{L}\right] .
$$

The longitudinal magnetization density is $\delta m_{x}^{i}=0.5\left(\delta m_{\perp}^{i}\right)^{2} /\left(M_{0} v\right)=0.5 \theta^{2} M_{0} / v$ where $v$ is the atomic volume, $\theta=\delta m^{i} / M_{0}$ is the precession angle. Hence

$$
\Delta V=0.125 e \theta^{2} M_{0} /\left(v \mu_{B}\right) f\left[\left(\xi^{L}-1\right)\left(1 / N_{+}^{L}+2 l_{1} /\left(\lambda N^{R}\right)+1 / N_{-}^{L}\right)+1 / N_{+}^{L}-1 / N_{-}^{L}\right] .
$$

As expected, this d.c. voltage is proportional to $\theta^{2}$, as is observed experimentally. Most importantly, it is proportional only to a ratio of the conductances. Hence its magnitude is not small. The factor f, as given after eq. (18), depends on the asymmetry between the majority spin and the minority spin conductances in the insulator. The larger the difference, the larger the value of $|f|$. We next estimate the order of magnitude of DeltaV.

We expect $M_{0} /\left(v \mu_{B}\right)$ to be of the order unity, $e / N(N$ is the average density of states ) to be of the order of 0.1 volt. Dependening on the asymmetry between the majority and the minority spin tunnel conductances in the insulator, the value of $\mathrm{f}$ can range between 1 and 0.1. Similarly, depending on the asymmetry between the majority and the minority spin 
conductances in the ferromagnet $\xi^{L}-1$ can range in value between 1 and $0.1 ; 1 / N_{+}-1 / N_{-}$ to be of the order of $1 / N$ to $0.1 / N$. Hence $\Delta V \approx\left(10^{-2}-10^{-4}\right) \theta^{2}$ volt. For $\theta \approx 0.1, \Delta V \approx$ $\left(10^{-4}-10^{-6}\right)$ volt, in agreement with the experimental results, which is larger than microvolts. We next address the issue of damping.

Damping: The loss can come from three sources: (1) from the interface inhomogeneity, (2) from loss of the transverse magnetization current through the barrier, (3) from loss of the longitudinal magnetization current. As we explain below, these contributions have different dependence on the external RF magnetic field. The contributions for the first two sources to the damping coefficient are independent of the field strength; that from the last source is proportional to the input power. The contributions from the last two sources are inversely proportional to the junction resistance and thus are much smaller for tunnel junctions.

We first estimate the loss connected with the longitudinal magnetization. This loss is equal to $\sum_{s} j_{s}^{2} r_{s}$ where $j_{s}, r_{s}$ is the current and junction resistance for spin s. This is of the order of $\left(\delta m_{x}^{i}\right)^{2}|T|^{2}$. Since $\delta m_{x}$ is proportional to the input power, this loss is proportional to the power squared. Its contribution to the damping coefficient is obtained by normalizing the loss by the energy density and hence is proportional to the power. Because this loss is proportional to $|T|^{2}$ its contribution is small for tunnel junctions. Similarly, we expect the transverse magnetization current to incur a loss of the order of $\left(\delta m_{\perp}^{i}\right)^{2}|T|^{2}$. Since $\delta m_{\perp}$ is proportional to the field, this loss is propotional to the power. Its contribution to the damping coefficient, again obtained by normalizing with espect to the energy density, is thus independent of the power. This loss is also proportional to $|T|^{2}$ and will be small for tunnel junctions.

We next estimate the loss connected with the interface inhomogenity. This requires knowledge of $\delta M_{30,40}$ which we now determine. Again, we expect the transverse magnetization to be a sum of a term that is the solution of the inhomogeneous equation $\left(\delta m^{i}\right)$ and terms that are solutions of the homogeneous equation $\left(\delta M_{3,4}\right)$. We calculate $\delta M_{3,4}$ using the boundary condition given by eq. (7). From eq. (15) the transverse magnetization current at the boundary is

$$
\hat{\mathbf{J}}_{\mathrm{M}}^{L}=-\frac{D_{M}^{\prime}}{l_{3}} \mathbf{e}_{+} \delta M_{30}^{L}-\frac{D_{M}^{\prime}}{l_{4}} \mathbf{e}_{-} \delta M_{40}^{L}
$$

The pseudo spin current in eq. (7) is thus given by

$$
\hat{\mathbf{I}}_{\mathrm{M}}^{L}=-\left(D_{M}^{\prime}+i \gamma J M_{0}\right) \delta M_{30}^{L} \mathbf{e}_{+} / l_{3}-\left(D_{M}^{\prime}-i \gamma J M_{0}\right) \mathbf{e}_{-} \delta M_{40}^{L} / l_{4}
$$


Eq. (7) also involves the tunnelling transverse current $J_{m}^{t}$. To evaluate this we follow standard practice [15] and calculate the rate of change of the transverse magnetization due to tunnelling. We found that the tunnelling current for the transverse magnetization can be written as $J_{M+}^{t}=M_{+}^{L}\left(g_{1}+i g_{2}\right)+M_{+}^{R}\left(g_{3}+i g_{4}\right)$ where[16] $g_{1,2,3,4}$ are proportional to $|T|^{2}$. A similar equation for $J_{M-}$ can be written down. This shows that the contribution from the tunnelling current is smaller than the other terms in eq.(7) and thus will be treated by perturbation theory. We finally obtain to lowest order $-I_{\mathbf{M}}^{L}=\gamma K_{s} \mathbf{M}_{0} \times\left(\delta \mathbf{m}^{i}+\delta \mathbf{M}_{0 s}\right)$. Substituing in the expression for $I_{m}$, this equation becomes

$$
\left[ \pm i D_{M}^{\prime} /\left(l_{ \pm} \gamma K_{s} M_{0}\right)-1-J /\left(l_{ \pm} K_{s}\right)\right] \delta M_{ \pm 0}^{L}=\delta m_{ \pm}^{i}
$$

Here $l_{+}=l_{3}, l_{-}=l_{4}, \delta M_{+}=\delta M_{30}, \delta M_{-}=\delta M_{40}$. As we go away from the interface, the transverse magnetization density dies off exponentially. The total magnetization is given by $\delta M_{ \pm} l_{ \pm}=\delta m_{ \pm}^{i} / X_{ \pm}$where $X_{ \pm}=\left[ \pm i D_{M}^{\prime} /\left(\gamma K_{s} M_{0}\right)-l_{ \pm}-J / K_{s}\right]$. The correction term due to the tunnelling magnetization current is equal to $-X^{-1} I_{m}^{t} /\left(\gamma K_{s} M_{0}\right)$.

The magnetic susceptibility, given by $\left(\delta M_{ \pm}^{L} l / d_{F}+\delta m_{ \pm}\right) / H_{1, \pm}$, becomes $\chi=\chi^{0}(1+$ $\left.X^{-1} / d_{F}\right)$. The additional damping comes from the imaginary part of $\chi$ which now contains a term proportional to $\operatorname{Re}\left(\chi^{0}\right) \operatorname{Im} X^{-1} / d_{F}$. This term is proportional to the metallic "resistance" $D_{M}^{\prime}$ which in turn comes from the spatially varying part of the magnetization induced by the surface, as we have anticipated. This contribution is not a function of the junction resistance and will be of the same order of magnitude for multilayers as well as for tunnel barriers.

In conclusion, we discussed in this paper the voltage and the damping of an RF field in ferromagnetic tunnel junctions. The voltage is controlled by changes of the longitudinal magnetization whereas the damping seems mainly associated with the transverse magnetization. Additional sources that can induce transverse magnetization localized near the interface can come from localized changes of the Hamiltonian such as the surface anisotropy. The calculation in this paper can be trivially extended to junctions with ferromagnets on both sides. For junctions involving two ferromagnets on opposite sides (F1-I-F2 or F1-F2), the interface anisotropy $K_{s}$ will contain a term from the dipolar interaction between F1 and F2. The loss will then be a function of the orientation of the magnetizations of F1 and F2, consistent with experimental results. 
STC is supported in part by the DOE. We thank John Xiao for helpful conversation.

[1] M. Ziese and M.J. Thornton, Spin Electronics (Springer, Berlin 2001).

[2] L. Berger, Phys. Rev. B 54, 9353 (1996).

[3] J.C. Slonczewski, Phys. Rev. B39, 6995 (1989), Jour. Magn. Magn. Mater. 159, L1 (1996); ibid., 195, L261 (1999).

[4] J.A. Katine et al. Phys. Rev. Lett. 84, 3149 (2000); F.J. Albert, J.A. Katine, R.A. Buhrman and D.C. Ralph, Appl. Phys. Lett. 77, 3809 (2000); S.J.C.H. Theeuwen et al. Appl. Phys. Lett. 75, 3677 (1999); E.B. Myers, D.C. Ralph, J.A. Katine, R.N. Louie, and R.A. Buhrman, Science 285, 867 (1999).

[5] M. Tsoi et al., Phys. Rev. Lett. 80, 4281 (1998); Nature 406, 46 (2000).

[6] Y. Tserkovnyak, A. Brataas, and G. E. W. Bauer, Phys. Rev. Lett, 88 117601, (2002). X. Wang, G. E. W. Bauer, B. J. v. Wees, A. Brataas, and Y. Tserkovnyak, Phys. Rev. Lett. 97, $216602(2006)$.

[7] S. Mizukami, Y. Ando, and T. Miyazaki, J. Magn. Magn. Mater. 226-230, 1640 (2001); B. Heinrich, Y. Tserkovnyak, G. Woltersdorf, A. Brataas, R. Urban, and G. E. W. Bauer, Phys. Rev. Lett. 90, 187601 (2003).

[8] M. V. Costache, M. Sladkov, S. M. Watts, C. H. van der Wal, and B. J. van Wees, Phys. Rev. Lett. 97, 216603 (2006).

[9] S. T. Chui and J. Cullen, Phys. Rev. Lett 74, 2118 (1995); S. T. Chui and L. Hu, Appl. Phys. Lett. 80, 273 (2002); S. T. Chui, US patent no. 5757056 (1998).

[10] M. Johnson and R. H. Silsbee, Phys. Rev. Lett. 55, 1790 (1985); P. C. van Son, H. Van Kempen and P. Wyder, Phys. Rev. Lett. 58, 2271 (1987).

[11] T. Moriyama, R. Cao, X. Fan, B. K. Nikolic, G. Xuan, and J. Kolodzey, and John Q. Xiao, unpublished.

[12] S. Englesberg and W. F. Brinkman, Phys. Rev. Lett. 21, 1187 (1968); D. Langreth and J. W. Wilkins, Phys. Rev. B6, 3189 (1971).

[13] Z. F. Lin and S. T. Chui, Phys. Lett. A 332, 115 (2004).

[14] See Ya. B. Bazaliy, B. A. Jones and Shou-Cheng Zhang, Phys. Rev. B57, R3213 (1998) for a description in the context of the Hubbard model. 
[15] G. D. Mahan, "Many Particle Physics", 3rd Ed., p. 561 Kluwer Academic, New York, (2000) [16] $g_{1}=\pi \sum-\left|T_{--}\right|^{2} \delta\left(-e_{L-}+e_{R-}\right)-\left|T_{++}\right|^{2} \delta\left(-e_{L-}+e_{R+}\right), g_{2}=\sum\left(-\left|T_{--}\right|^{2}+\left|T_{++}\right|^{2}\right) /\left(-i e_{R+}+\right.$ $\left.i e_{L-}\right), g_{3}=\sum T_{++} T_{--}^{*} \pi\left(\delta\left(e_{L-}-e_{R-}\right)++\delta\left(e_{L+}-e_{R+}\right), g_{4}=\sum T_{++} T_{--}^{*}\left[1 /\left(i e_{L-}-i e_{R}\right)+\right.\right.$ $\left.1 /\left(i e_{R}-i e_{L+}\right)\right]$ 